\title{
Special issue on big data for IoT cloud computing convergence
}

\author{
Z.X. Hou ${ }^{\mathrm{a}}$ and Y. Xiao ${ }^{\mathrm{b}}$ \\ ${ }^{a}$ Changsha University of Science and Technology, China \\ ${ }^{\mathrm{b}}$ University of Alabama, USA
}

Big data refers to the data set that cannot be captured, managed and processed by conventional software tools within a certain period of time. It is a massive, high growth rate and diversified information asset that requires new processing patterns to have stronger decision-making power, insight and process optimization capabilities The last decade has seen huge advances in the scale of data we routinely generate and collect in pretty much everything we do, as well as our ability to use technology to analyze and understand it. We have been witnessing a digital revolution associated with developments of various emerging technologies including ubiquitous computing devices, sensors and sensing devices, smart devices, cloud computing and big data analytics tools are dramatically changing the mode and accessibility of science, research and practice in all domains $[1,4,8]$.

The Internet of Things is an important part of the new generation of information technology, and also an important stage of development in the "information" era. Internet of Things (IoT) applications are considered to be a major source of big data obtained from a more connected dynamic and real life world and is evolving at a rapid pace. The realization of the IoT vision brings Information and Communication Technology (ICT) closer to many aspects of the real-world life instead of the virtual life through advanced theories, algorithms and applications. Technology of real-world IoT based on cloud computing has rapidly emerged as a novel industry and life paradigm. These topics will be the most comprehensive field focused on the various aspects of advances in computer engineering technologies, applications, and services. In cloud computing environments that include mobile infrastructures, the most important and final goal is to provide users more secure and richer Internet of Things ser- vices. Tremendous advances in algorithms of sensing, processing, communication and actuating core technologies are leading to new intelligent IoT services in our life such as smart cities, smart healthcare, smart grids, and others to improve all aspects of life. There might be many issues to realize it and provide intelligence IoT services based on the advanced applied algorithms and application technologies with much effort and enormous attention. The advanced applied algorithms and application technologies of this research area poses challenges such as context information fusion, security, reliability, autonomous and intelligent connecting, trust application and framework for realworld life. Advanced algorithms and applications for IoT based on the cloud computing research contributions that present new technologies, concepts, or analysis, reports on experiences and experiments of implementation and application of theories, and tutorials on new trends, are required in this research fields [9].

The following is a non-exhaustive list of topics in focus of this special issue:

- Interoperable and interactive middleware for IoT on cloud computing

- Advanced IoT services algorithms, technologies and applications on cloud computing

- Real-time algorithms, technologies and applications with real-world IoT on cloud computing

- Related theory and technologies between web service and IoT in cloud computing

- Advanced theory and technologies for High Performance and Communications with IoT

- Clustering with Evolutionary and Swarm Algorithms for Big Data Analytics

- Cloud computing and big data analytics

- Data mining and machine learning for IoT 
- Big data sensing and IoT frameworks and infrastructures

This special issue is dedicated to exploring recent advances in big data for IoT cloud computing convergence. It is based on papers submitted to and presented at the 2018 International Conference on Robots \& Intelligent System (ICRIS2018) held in May 26-27 at Changsha, China. Papers were selected using the following criteria:

- Quality and originality in theory and methodology;

- Relevance to big data for IoT cloud computing;

- Application-oriented papers exhibiting originality with reasonable theoretical content;

- The novelty of theory and application focusing on big data for IoT cloud computing.

The papers that were eventually selected represent and characterize the outstanding and active studies in the area of big data for IoT cloud computing convergence and illustrate clearly the advantages of developing big data for IoT cloud computing convergence.

In the first paper [6], Xiaofeng Han, Liejun Wang and Jiwei Qin Tao provide a new indicator, which considers one of the non-functional attributes of web services-availability into the WSC. According to the service invocation structures, the global availability of the combined service can be obtained from the availability of a single web service. Results show that new method is reasonable and efficient compared with other counterparts.

In the second paper [5], for the problem of static scheduling about related tasks in cloud environment, Xiaozhong Geng, Lan Yu, Jie Bao and Geji Fu provides a new task scheduling algorithm. The experimental results display the influence of the number of tasks on the performance of the algorithm when CCR is different. Compared with HNDP and HEFT, the new algorithm has a smaller SLR value. Moreover, with the increase of CCR, the advantages of the new algorithm are obvious.

In the third paper [3], Bin Dai provides a new design method of complex wind power generation parameter control system based on embedded control combined with Internet of Things. In this method, in the Internet of Things environment, the overall model of the system is determined, and embedded control for big data of the complex wind power generation parameters is performed to achieve adaptive fusion of control instruction information, and optimize the big data transmis- sion and scheduling of control instruction sets. Test results show that the complex wind power generation parameter control system designed through this method has relatively strong parameter data control processing capability, good stability of control instruction transmission and high parallel processing capability of control instruction sets, and this method improves control quality of the overall system.

In the fourth paper [2], in order to improve the intelligence of higher mathematics teaching resource scheduling, Dr Tiejun Chen provided a higher mathematics teaching resource scheduling system based on cloud computing. A balanced modulation control algorithm for higher mathematics teaching resources is proposed on the overall design of this system and an association rule constraint model for higher mathematics teaching resource allocation is constructed based the design results. The simulation results show that, in the allocation of teaching resources, the balance coefficient of higher mathematics teaching resource scheduling system based on cloud computing has been increased by $12.54 \%$, and the accuracy of sending and receiving teaching data provided by this system has been improved by $13.6 \%$, which indicating that this system has relatively good intelligence and strong human-computer interaction ability.

In the fifth paper [10], Jian Shen, Haihang Jiang, Fei Yang and Zhenxing Yao provided a hybrid model composed of the correlation ratio analysis and the Support Vector Machine (SVM) for trip mode recognition. The correlation ratio analysis algorithm is applied to determine the optimal time window of the input attributes so as to optimize the input parameters, and the SVM is applied to carry out the trip mode recognition for the whole trip. Its effectiveness is proved by experiment and these results provide a reference for using the smartphone sensor datasets to supplement or even replace household travel surveys in transportation planning in the future.

In the sixth paper [7], Hua Liang and Feng Zeng firstly analyze the shortcomings of node based on node quantization, then propose a data forwarding mechanism based on node function and node centricity, and gets its performance improved effectively, and reduces the energy consumption of nodes.

In the seventh paper [11], in order to solve the frequent communication obstacle problem in MIMO system communication caused by routing conflicts, Kun Zhang, Chong Shen and Hanwen Li provided a fast optimal node locating algorithm for MIMO system communication based on link balance control. It shows that 
this algorithm can provide high accuracy in locating the optimal nodes for communication, and can ensure high locating efficiency, which effectively improves the communication transmission quality of MIMO system.

We very much hope that readers of this special issue will find the ideas presented in it of interest to them. We also hope that the material will be sufficiently stimulating to encourage many readers to explore the relationships between big data and IoT cloud computing further. This special issue is directed toward practicing engineers, researchers and industry operations managers, who wish to enhance or deepen their knowledge on the fusion of big data and IoT cloud computing. We do hope that these carefully selected papers will inspire and stimulate the industrial effort to use big data and IoT cloud computing techniques in their ITS applications.

\section{Acknowledgements}

The Guest Editors would like to express their appreciation to the authors for their high-quality and innovative work. Special thanks to the Editor, Yuefeng Li and Vijay V. Raghavan, for his considerable support and guidance, which made this special issue possible. In addition, the Guest Editors are deeply indebted to the many anonymous referees who performed their assignments in a highly professional manner.

\section{Conflict of interest}

No potential conflict of interest was reported by the authors.

\section{Funding}

The special issue is strongly supported by the $\mathrm{Na}$ tional Social Science Fund Project of China (No. 51678077).

\section{References}

[1] T. Akidau, R. Bradshaw, C. Chambers, S. Chernyak, R. Fernández-Moctezuma, R. Lax, S. McVeety, D. Mills, F. Perry, E. Schmidt and S. Whittle, The dataflow model: A practical approach to balancing correctness, latency, and cost in massive-scale, unbounded, out-of-order data processing, PVLDB 8(12) (2015), 1792-1803.

[2] T.J. Chen, Higher mathematics teaching resource scheduling system based on cloud computing, Web Intelligence 17(2) (2019). doi:10.3233/WEB-180379.

[3] B. Dai, Design of complex wind power generation parameter control system based on embedded control combined with Internet of Things, Web Intelligence 17(2) (2019).

[4] J. Dean and S. Ghemawat, MapReduce: Simplified data processing on large clusters, in: OSDI, 2004, pp. 137-150.

[5] X.Z. Geng, L. Yu, J. Bao and G.J. Fu, A task scheduling algorithm based on priority list and task duplication in cloud computing environment, Web Intelligence 17(2) (2019).

[6] X.F. Han, L.J. Wang and J.W. Qin, Combine availability with user preferences for efficient WSC, Web Intelligence 17(2) (2019).

[7] H. Liang and F. Zeng, Data forwarding mechanism based on node function and node centricity, Web Intelligence 17(2) (2019).

[8] X.F. Meng and C. Xiang, Big data management: Concepts, techniques and challenges, Journal of Computer Research and Development 50(1) (2013), 146-169.

[9] M. Shah, Big data and the Internet of things, in: Big Data Analysis: New Algorithms for a New Society, 2016, pp. 207-237. doi:10.1007/978-3-319-26989-4_9.

[10] J. Shen, H.H. Jiang, F. Yang and Z.X. Yao, Trip mode recognition using smartphone sensor data under different sampling frequencies, Web Intelligence 17(2) (2019).

[11] K. Zhang, C. Shen and H.W. Li, A fast optimal node locating algorithm for MIMO system communication based on link balance control, Web Intelligence 17(2) (2019). 